\title{
Asymptomatic Pneumoperitoneum After Endoscopic Treatment of Pseudocysts
}

\begin{abstract}
4 26-year-old woman with alcohol abuse presented with comlaints of abdominal distress and weight loss during the previous our months. The physical examination revealed malnutrition, iscites, painful abdomen, and an epigastric mass. The laboratory ests showed elevated amylase, lipase, alkaline phosphatase, gslutamyl transpeptidase and low hemoglobin $(10.2 \mathrm{~g} / \mathrm{dl})$ levels Endoscopy showed esophageal varices and compression of the zastric wall. Computed tomography (CT) revealed ascites, a lilated pancreatic duct, small cysts, and a $15-\mathrm{cm}$ cyst in the body ind tail of the pancreas (Figure 1). She was placed on a nothingsy-mouth regimen with parenteral nutrition, and treatment with ;omatostatin, fluids, and electrolytes was commenced. She eceived antibiotics, and was referred for endoscopic pseudocyst trainage.
\end{abstract}

We first carried out a cystogastrostomy, with placement of a 7-Fr ;tent $(1,2)$; a brownish fluid was drained (amylase $12640 \mathrm{U} / 1$ ) staphylococcus aureus was cultured. Endoscopic retrograde cholingiopancreatography (ERCP) was carried out 14 days later, and a 7 - Fr, 10-cm transpapillary pancreatic stent was placed to drain the ther cysts. A post-ERCP CT showed improvement of the pseudocysts, and also a pneumoperitoneum (Figure 2). Despite the CT findings, she was asymptomatic and continued to eat, and is still feeling well.

Pseudocysts in chronic pancreatitis may last for long periods, and some should be treated (2). Those larger than $6 \mathrm{~cm}$ usually persist, and may lead to complications such as infection, abscess, hemorrhage, rupture, and intestinal or biliary obstruction (3). Endoscopic drainage is an effective treatment, with low mortality and morbidity rates. Pseudocysts that do not communicate with the main pancreatic duct are drained by cystoenterostomy $(1,2)$; transpapillary stents are placed in communicating cysts (4). Complications that have been described previously include mild pancreatitis, stent occlusion, and infection, but not pneumoperitoneum with a favorable outcome after conservative treatment. The pneumoperitoneum is thought to have been caused by the development of a fistula after the cystogastrostomy, allowing air from the stomach to enter the peritoneal cavity. Endoscopic therapy is safe and effective, and should be the primary choice for the drainage of pancreatic pseudocysts. 


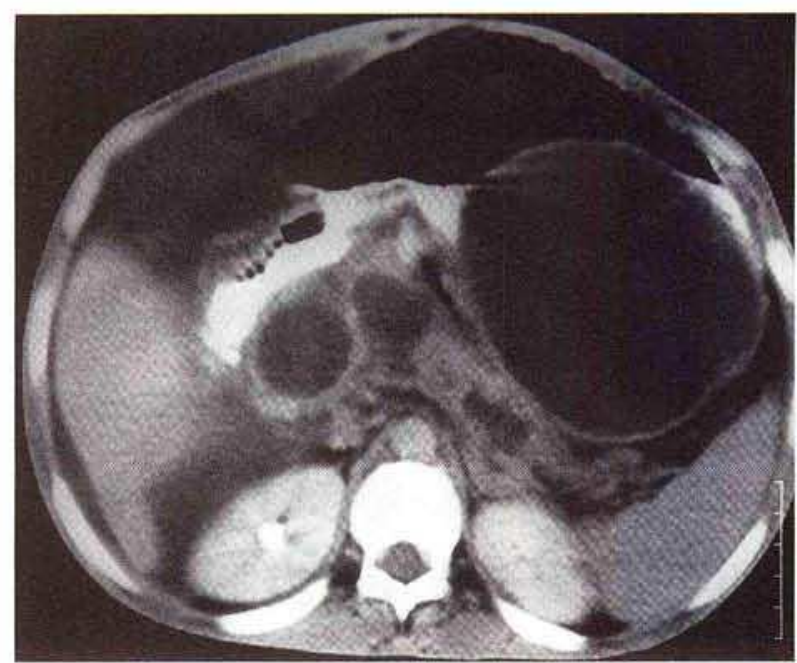

Figure 1: $C T$, showing the dilated main pancreatic duct and cysts in the pancreatic head and body. A large pseudocyst $(15 \mathrm{~cm})$ is present in the body and tail of the pancreas.

\section{E. Della Libera, A. P Ferrari, Jr.}

Division of Gastroenterology, São Paolo School of Medicine, São Paolo, Brazil

\section{References}

1. Cremer M, Devière J, Engelholm L. Endoscopic management of cysts and pseudocysts in chronic pancreatitis: long-term follow-up after 7 years of experience. Gastrointest Endosc 1989; 35: 1-9.

2. Sahel J. Endoscopic drainage of pancreatic cysts. Endoscopy 1991; 23: 181-4.

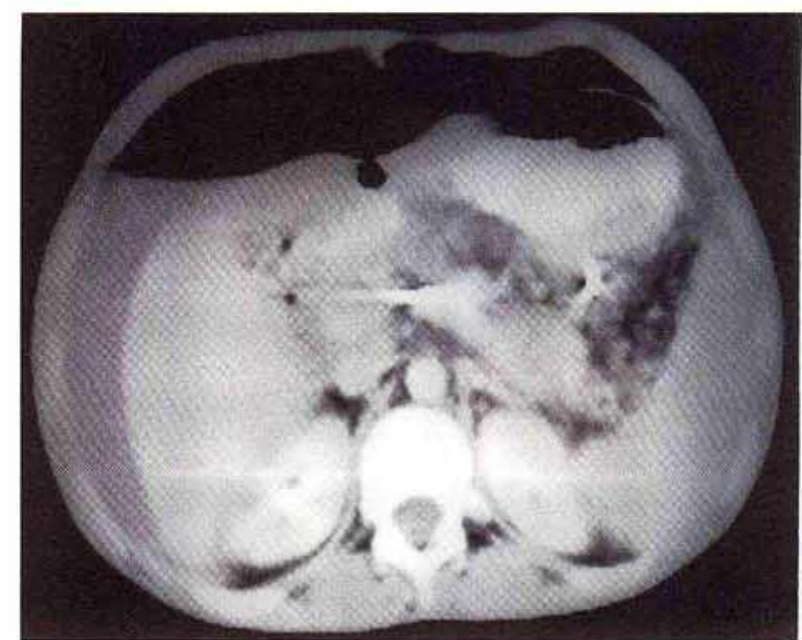

Figure 2: CT after the second ERCP, showing the transpapillary anc cystogastrostomy stents and the pneumoperitoneum. The pseudocysts have been almost completely drained.

3. Bradley EL III, Clements JL Jr, Gonzales AC. The natural history of pancreatic pseudocysts: a unified concept of management. Am J Surg 1979; 137: 135-41.

4. Kozarek RA, Ball TJ, Patterson DJ. Endoscopic transpapillary therapy for disrupted pancreatic duct and peripancreatic fluic collections. Gastroenterology 1991; 100: 1362-70.

\section{Corresponding Author}

A. P. Ferrari, Jr., M.D., R. Machado Bittencourt, 379 apt. 91 São Paulo CEP 04044-001, Brazil , Fax: + 55-11-575-3834 